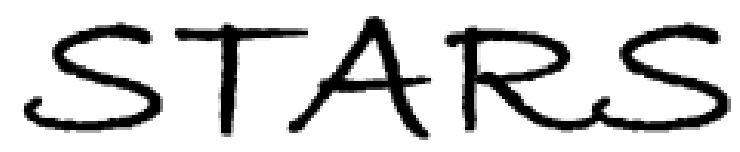

University of Central Florida

STARS

$1-1-2014$

\title{
Optical Salisbury screen with design-tunable resonant absorption bands
}

Janardan Nath

University of Central Florida

Evan Smith

University of Central Florida

Douglas Maukonen

University of Central Florida

Robert E. Peale

University of Central Florida

Find similar works at: https://stars.library.ucf.edu/facultybib2010 University of Central Florida Libraries http://library.ucf.edu

This Article is brought to you for free and open access by the Faculty Bibliography at STARS. It has been accepted for inclusion in Faculty Bibliography 2010 s by an authorized administrator of STARS. For more information, please contactSTARS@ucf.edu.

\section{Recommended Citation}

Nath, Janardan; Smith, Evan; Maukonen, Douglas; and Peale, Robert E., "Optical Salisbury screen with design-tunable resonant absorption bands" (2014). Faculty Bibliography 2010 s. 5877.

https://stars.library.ucf.edu/facultybib2010/5877

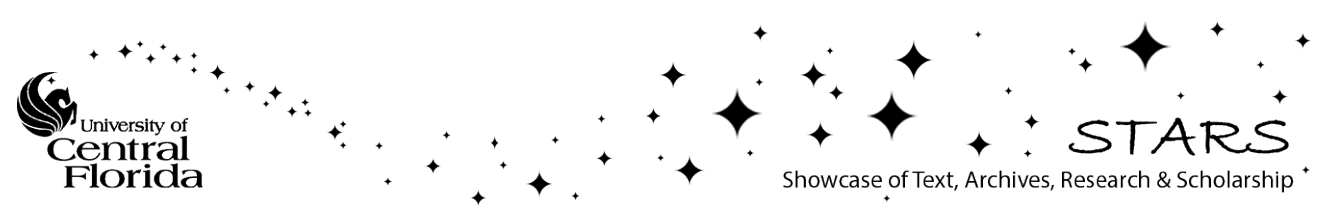




\section{Optical Salisbury screen with design-tunable resonant absorption bands}

Cite as: J. Appl. Phys. 115, 193103 (2014); https://doi.org/10.1063/1.4876117

Submitted: 23 January 2014 . Accepted: 30 April 2014 . Published Online: 15 May 2014

Janardan Nath, Evan Smith, Douglas Maukonen, and Robert E. Peale

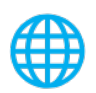

\section{ARTICLES YOU MAY BE INTERESTED IN}

Graphene based salisbury screen for terahertz absorber

Applied Physics Letters 104, 081106 (2014); https://doi.org/10.1063/1.4866665

Ultra-thin perfect absorber employing a tunable phase change material

Applied Physics Letters 101, 221101 (2012); https://doi.org/10.1063/1.4767646

Enhanced universal absorption of graphene in a Salisbury screen

Journal of Applied Physics 121, 023110 (2017); https://doi.org/10.1063/1.4973898

\section{Applied Physics Reviews} Now accepting original research

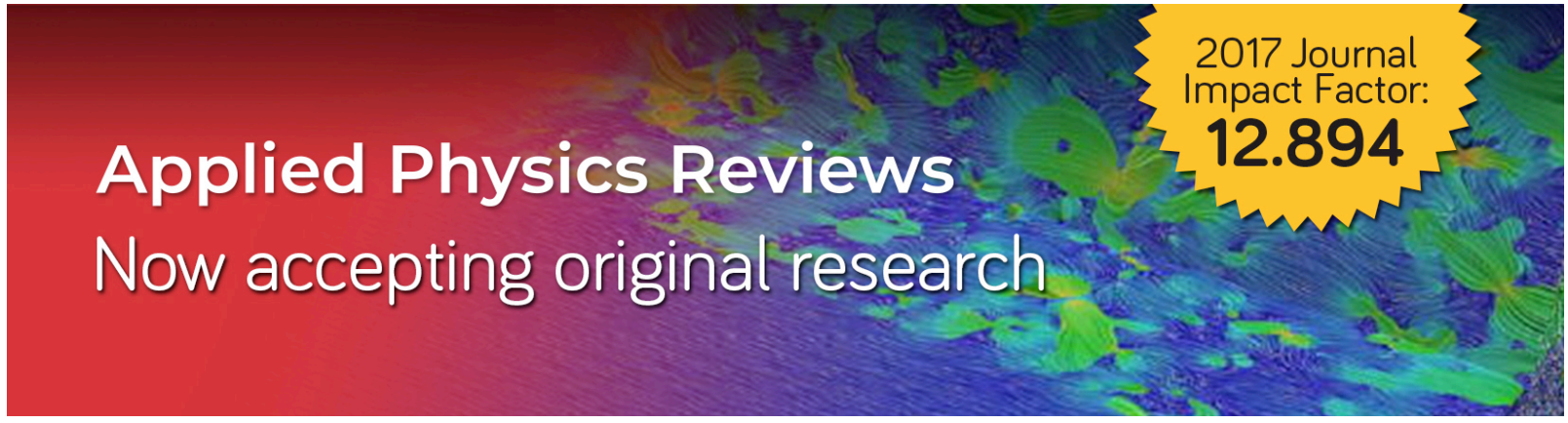




\title{
Optical Salisbury screen with design-tunable resonant absorption bands
}

\author{
Janardan Nath, Evan Smith, Douglas Maukonen, and Robert E. Peale ${ }^{\text {a) }}$ \\ Department of Physics, University of Central Florida, 4000 Central Florida Blvd, Orlando, Florida 32816, \\ USA
}

(Received 23 January 2014; accepted 30 April 2014; published online 15 May 2014)

\begin{abstract}
A thin-film selective absorber at visible and near infra-red wavelengths is demonstrated. The structure consists of an optically thick layer of gold, a $\mathrm{SiO}_{2}$ dielectric spacer and a partially transparent gold film on top. The optical cavity so formed traps and absorbs light at a resonance wavelength determined by the film thicknesses. Observed fundamental-resonance absorption strengths are in the range 93\%-97\%. The absorption red-shifts and broadens as the thickness of the top gold layer is decreased with little change in absorption strength. Thus, strong absorption with design-tunable wavelength and width is achieved easily by unstructured blanket depositions. Observed angle-dependent spectra agree well with the recent three-layer analytical model of Shu et al. [Opt. Express 21, 25307 (2013)], if effective medium approximation is used to calculate the permittivity of the top gold film when it becomes discontinuous at the lowest thicknesses. C 2014 AIP Publishing LLC. [http://dx.doi.org/10.1063/1.4876117]
\end{abstract}

\section{INTRODUCTION}

Wavelength-selective plasmonic and metamaterial perfect absorbers have been studied extensively for various applications including infra-red (IR) bolometers, thermal imaging, thermal emitters, plasmonic sensing, and solar cells. ${ }^{1-14}$ Up to $\sim 99 \%$ absorption is reported experimentally in the visible, near-IR (NIR), mid-IR, and at terahertz frequencies. ${ }^{4-14}$ However, the emphasis has been on threedimensional structures, which depend on numerical design and sophisticated fabrication techniques, such as electron beam lithography for in-plane patterning. Particularly, in the visible and near-IR, nanoscale structures such as periodic nano-discs, ${ }^{4}$ squares, ${ }^{5-7}$ metallic gratings and trapezoids, ${ }^{8}$ and split ring resonators $(\mathrm{SRRs})^{9}$ have been investigated.

This paper presents experimental characterization of an efficient selective absorber formed by three simple layers without any in-plane patterning at all. Such triple-layer structure composed of conductor-dielectric-conductor is known as a "Salisbury screen" at radio wavelengths and has been used to reduce radar cross-sections for military applications. ${ }^{15,16}$ Adaptation of this technology to visible and nearIR wavelengths has been investigated theoretically by Shu et al. ${ }^{17}$ Unlike metamaterial absorbers, the device consists of unpatterned thin metal and dielectric films, which can be fabricated by any suitable means of blanket deposition. On the other hand, a possible disadvantage is that this type of absorber is 3-10 times thicker than previously reported metamaterial absorbers. ${ }^{4-7}$ In principle, freedom from lithographic patterning enables application to arbitrarily large areas by wet chemical methods. The resonant absorption occurs in multiple bands because of light trapping in the Fabry-Perot cavity so formed. The resonance wavelength depends on the thicknesses of the dielectric spacer and the

\footnotetext{
a) Author to whom correspondence should be addressed. Electronic mail: robert.peale@ucf.edu.
}

top metal, while the deposited thickness of the latter strongly controls the resonance width without significantly affecting the absorption strength.

One of the primary objectives of this paper is to experimentally test the predictions of the analytic theory presented recently by Shu et al. ${ }^{17}$ When the deposited thickness of our top-layer metallization is at least $20 \mathrm{~nm}$, the film is more or less continuous, and theory agrees fairly well with experiment. On the other hand, for thicknesses much below $20 \mathrm{~nm}$, the discontinuities in our films become significant, such that the theory of Ref. 17 is inapplicable. In fact, experiment then differs very strongly from that theory.

A second objective of this paper is to extend the theory of Ref. 17 to the case of discontinuous top metal films. Our hypothesis is that the theory will work if the discontinuities are smaller than the wavelength, such that macroscopic electrodynamics still applies, with suitable optical constants. We find that use of permittivity values obtained from effective medium approximation (EMA $)^{18-20}$ restores the substantial agreement with experiment, especially with respect to the wavelengths of the resonances, their widths, and their peak absorption.

For applications, there has been significant effort to broaden the absorption in resonant plasmonic/metamaterial nano-structures. ${ }^{6,8,9}$ Reference 6 used a multiplexed periodic checkerboard of metal squares to achieve $\sim 200 \mathrm{~nm}$ absorption band width at $3.45 \mu \mathrm{m}$ wavelength. Reference 8 employed crossed gratings and trapezoid structure to achieve $250 \mathrm{~nm}$ band width in the visible. Reference 9 investigated SRRs to realize $\sim 800 \mathrm{~nm}$ band width at $1.3 \mu \mathrm{m}$ wavelength. All structures required electron beam lithography, which is unsuited for large areas or mass production.

A non-lithographic approach to broad absorption was presented in Ref. 10, where nano-composite $\mathrm{SiO}_{2} / \mathrm{Au}$ films were co-evaporated. Strong absorption was achieved from 400 to $800 \mathrm{~nm}$ wavelength. However, the design-tuning range realized by changing the volume fraction of metal was only $\sim 100 \mathrm{~nm}$. 
In contrast to the metamaterial and composite approaches, the simple three-layer Salisbury screen presented here provides a wide range of possible center wavelengths and absorption widths, simply by changing the thicknesses of the depositions. Yet all investigated variations maintain a peak fundamental absorption of at least $93 \%$ and up to $97 \%$. No sophisticated lithography is required. In principle, low-cost wet chemical deposition could replace vacuum methods to achieve large area coverage.

\section{THEORY}

Fig. 1 presents a schematic of the 3-layered metaldielectric-metal absorber, whose reflectance is ${ }^{17}$

$$
R=\left|\frac{1}{r_{01}}+\frac{r_{01}-\frac{1}{r_{01}}}{\frac{r_{01}}{\phi}+1}\right|^{2},
$$

where

$$
\phi=\frac{1-r_{12}^{2} e^{2 i k_{2 z} d_{2}}}{r_{12}\left(1-e^{2 i k_{2 z} d_{2}}\right) e^{2 i k_{1 z} d_{1}}}
$$

and $r_{i, i+1}$ are the angle- and polarization-dependent Fresnel amplitude reflection coefficients for light travelling from medium $i$ to medium $i+1$. Thicknesses $d_{i}$ and relative permittivities $\varepsilon_{i}$ are as indicated in Fig. 1. The normal component of the wave vector in medium $i$ is

$$
k_{i z}=\frac{2 \pi}{\lambda} \sqrt{\varepsilon_{i}-\sin ^{2} \theta}
$$

where $\theta$ is the angle of incidence on the top layer and $\lambda$ is the free-space wavelength. Light that is partially reflected at the semitransparent medium 1 interferes with light that makes a round trip through medium 2 and is again transmitted by medium 1. The condition of destructive interference places a node at medium 1, which is the same condition as for resonant light trapping with multiple reflections inside the dielectric of a Fabry-Perot cavity. The trapped energy is consumed by losses in the three media, where the dissipated energy density is proportional to the frequency, to the imaginary part of the permittivity, and to the square of the amplitude of the electric field. ${ }^{21}$ This absorption mechanism for Fabry-

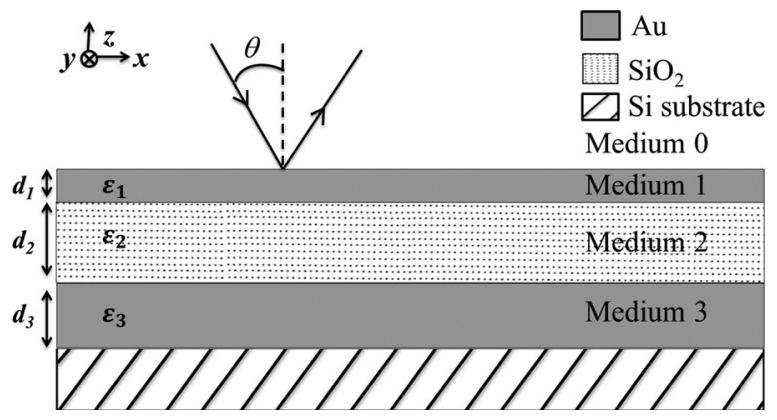

FIG. 1. Schematic diagram of the triple-layer metal-dielectric-metal absorber.
Perot type metamaterial absorbers has also been described by Hao et al. ${ }^{2}$

The theoretical absorption resonances red-shift, broaden, and weaken with decreasing top-layer thickness $d_{1}$. However, for deposited top layer thicknesses of $20 \mathrm{~nm}$ and below, the experimental shifts and broadening of the absorption bands greatly exceed those predicted by the theory, while the decrease in absorption strength is much less, if bulk permittivity values are assumed in the calculations. Such are inaccurate for very thin films, which become discontinuous. The observed length scale of the discontinuities is much less than our shortest wavelength (Fig. 2), so that macroscopic electrodynamics holds with an appropriately averaged permittivity, and we may neglect scattering.

The averaged permittivity is found by effective medium theory, ${ }^{18,19}$ where the Maxwell-Garnett approximation $(\mathrm{MGA})^{18}$ and Bruggeman $\mathrm{EMA}^{19}$ are the most commonly used formalisms. The latter is preferred for our films since MGA assumes a dilute system of non-interacting isolated nanoparticles. ${ }^{18,19}$ For normal incidence, we approximate our system as a 2-dimensional array of flat circular plates. The effective permittivity is then ${ }^{20}$

$$
\varepsilon_{e f f}=-s \pm \sqrt{s^{2}+\varepsilon_{A u}},
$$

where $s=\left(1+\varepsilon_{A u}\right) / 2+f\left(1-\varepsilon_{A u}\right)-1, f$ is the volume fraction of gold, $\varepsilon_{A u}$ is the complex frequency-dependent
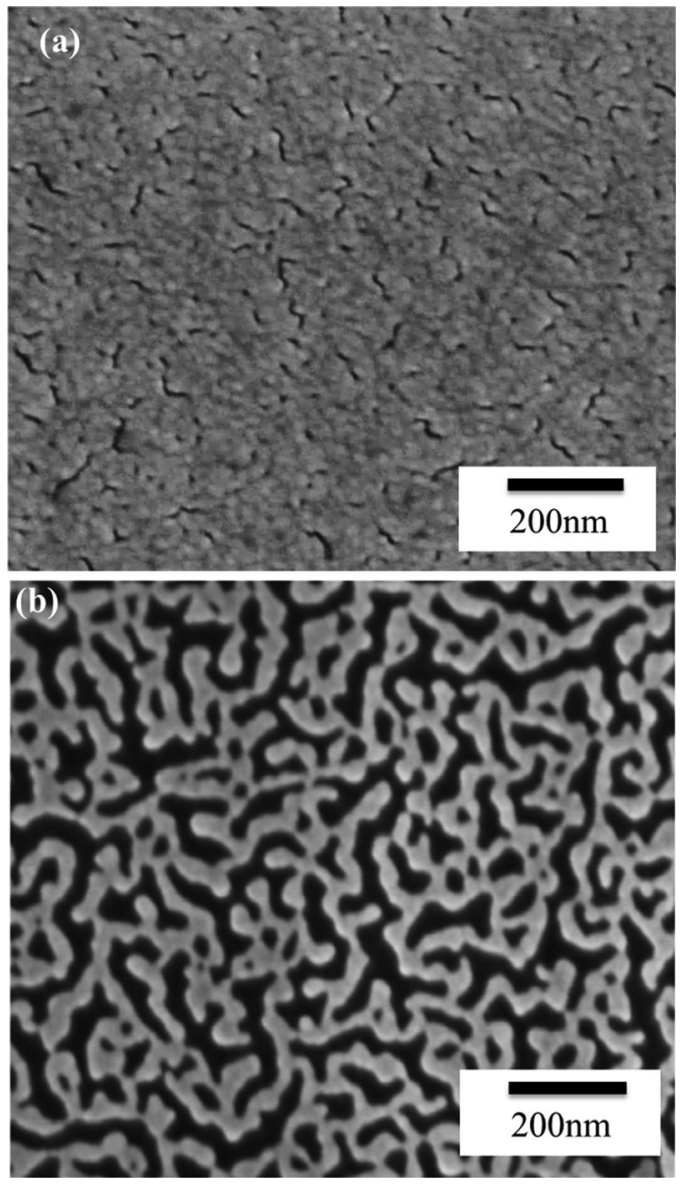

FIG. 2. SEM images of (a) 20- and (b) 10-nm-thick gold films. 
permittivity of bulk gold, and the permittivity of air has been taken to have the value unity. ${ }^{20}$ Positive or negative roots in Eq. (4) are chosen based on the physical requirement that $\operatorname{Im}\left(\varepsilon_{\text {eff }}\right)>0$ always. For $f>0.5, \operatorname{Re}\left(\varepsilon_{\text {eff }}\right)<0$, though its magnitude is smaller than that of the bulk metal. For, the films with $f=0.85$ and 0.54 studied here, over our spectral range, the $\operatorname{Re}\left(\varepsilon_{\text {eff }}\right)$ is smaller than for bulk by factors of $\sim 1.4$ and $\sim 25$ at $2.5 \mu \mathrm{m}$ wavelength, respectively. The $\operatorname{Im}\left(\varepsilon_{e f f}\right)$ is smaller than for bulk by factors of $\sim 1.5$ and $\sim 3$, respectively, except in the short wave part of the spectrum where it is actually larger than for bulk when $f=0.54$.

For non-normal incidence, the thickness of the metal becomes important due to shadowing of the open gaps in the film. This effect can be accounted for by increasing the gold fraction $f$ in the calculations. This is clear if one considers grazing incidence where the beam skims the top surface of the gold, while the open spaces remain hidden. A very simple 1D model of the decrease for rectangular gaps in their apparent volume caused by hard shadowing, which ignores the semi-transparency of gold at the thicknesses considered, gives

$$
f(\theta)=\left\{\begin{array}{l}
f_{0}+\frac{d_{1}\left(1-f_{0}\right) \tan \theta}{2 a}, \tan \theta<a / d_{1} \\
1-\frac{a\left(1-f_{0}\right)}{2 d_{1} \tan \theta}, \tan \theta>a / d_{1}
\end{array}\right.
$$

where $f_{0}$ is the apparent volume fraction at normal incidence and $a$ is the characteristic size of the gaps.

\section{EXPERIMENTAL DETAILS}

To fabricate devices, a $150 \mathrm{~nm}$ layer of gold was deposited by electron-beam evaporation on a $\mathrm{Si}$ substrate with a $10 \mathrm{~nm} \mathrm{Cr}$ sticking layer. $\mathrm{SiO}_{2}$ dielectric was deposited by plasma enhanced chemical vapor deposition (PECVD) from a liquid Tetraethyl-orthosilicate (TEOS) source. PECVD oxide and gold stick well without Cr. The thickness of the PECVD oxide film is determined from interference fringes measured in reflectance using a UV-Vis-NIR Cary500i spectrometer before the top metal deposition. The thin top layer of gold was deposited by DC sputtering, with thickness determined from the sputtering time, which was calibrated by step profilometry. Atomic Force Microscopy (AFM) (Digital Instruments Dimension 5000U) was used to characterize the final surface roughness of the structures. Specular reflectance at near normal incidence was measured using a BOMEM DA8 Fourier spectrometer with Si (visible) or InSb (near IR) detectors, quartz or $\mathrm{KBr}$ beam splitters, and quartz-halogen or globar sources, respectively. Angle dependent specular reflectance was measured using the Cary500i UV-Vis-NIR spectrometer. Reflectance $(R)$ is obtained by dividing the raw reflected power spectrum from the sample with that from a freshly evaporated, optically thick silver film on an optically polished flat. The initially focused incident beam of the spectrometer is collimated by a concave mirror and is incident on the sample at an angle of $8^{\circ}$ using a mirror assembly. Reflected light is finally diverged at the appropriate acceptance angle to the detector collection optic by another concave mirror. The absorptance $A=1-R$, since transmittance $T=0$ and we neglect scattering. The volume fraction of gold for the films, and characteristic dimension of the inhomogeneities, are determined from scanning electron microscope (SEM) images using imageJ software. $^{23}$

\section{RESULTS AND DISCUSSION}

Fig. 2 presents SEM images of two different preparations of the top gold film. Bright parts of the image are gold and dark parts are subsurface $\mathrm{SiO}_{2}$. As thickness decreases, the film becomes more and more discontinuous. ImageJ analysis of $20 \mathrm{~nm}$ thick gold films gives a value for the gold volume fraction $f=0.85 \pm 0.05$, where $f$ is determined by the ratio of extreme dark and bright areas of the image and the uncertainty comes from setting thresholds for brightness and contrast. For the $10 \mathrm{~nm}$ film, we find the area fraction $f=0.54 \pm 0.01$. The uncertainty is smaller here due to higher image contrast.

Fig. 3 compares experimental and theoretical (Eq. (1)) reflectance spectra, both for $8^{\circ}$ angle of incidence. Calculations performed at $0^{\circ}$ and $8^{\circ}$ angle of incidence are indistinguishable in comparison to the measurement uncertainties. Resonance peaks at these two angles differ by less than $0.003 \mu \mathrm{m}$ wavelength, or $0.27 \%$, for all sample conditions. The experimental spectrum was measured for an unpolarized beam, and the calculations plotted are an equally weighted average of TE and TM polarizations.

A number of trends are clear for calculated spectra that assume bulk permittivity values (dotted curves). ${ }^{22}$ As the oxide thickness $d_{2}$ is increased, the fundamental resonance red shifts, and a higher order resonance enters the spectral range.

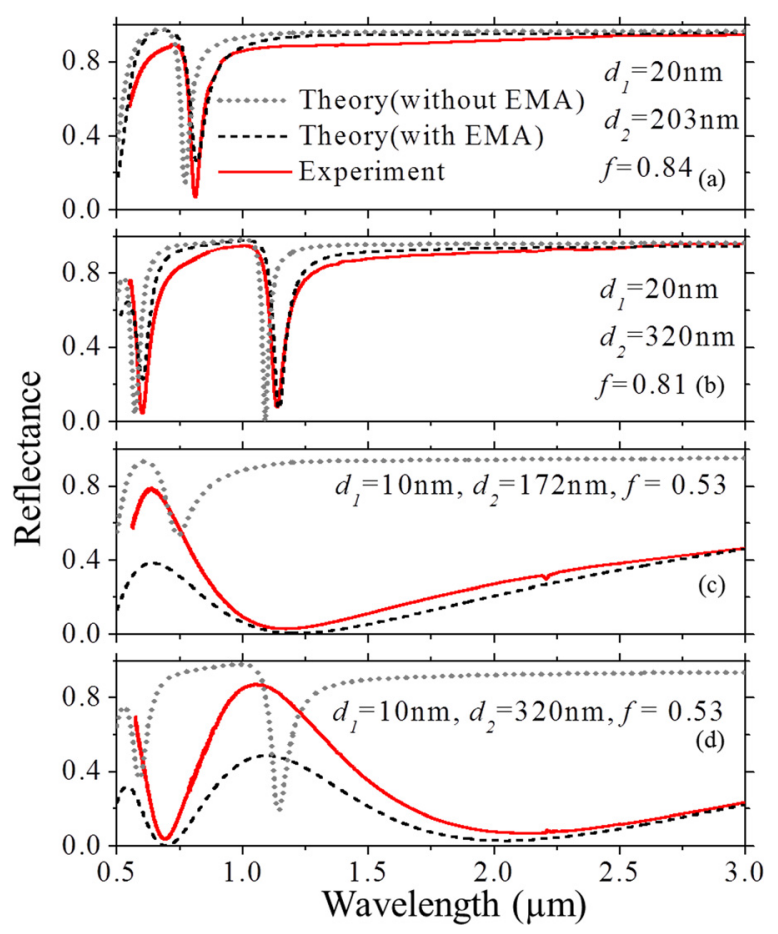

FIG. 3. Reflectance spectra for samples with $d_{1}$ and $d_{2}$ values as indicated. Calculations based on bulk or EMA permittivity values are compared to experiment for $8^{\circ}$ angle of incidence. 
The resonance line width is independent of the oxide thickness, but the depth of the fundamental is dependent. As top metal thickness $d_{1}$ decreases, the resonance red shifts and broadens modestly, and strength of absorption decreases significantly.

Figs. 3(a) and 3(b) compare reflectance spectra for absorbers with $d_{1}=20 \mathrm{~nm}$ and $d_{2}=203$ or $320 \mathrm{~nm}$, respectively. The positions and widths of the resonances calculated without EMA (black dotted line) agree poorly with experiment. The agreement is much better when the EMA permittivity is used (gray dotted lines) with $f=0.84$ and 0.81 for samples with $d_{2}=203 \mathrm{~nm}$ and $d_{2}=320 \mathrm{~nm}$, respectively. The $f$ values that gave best fit to the experimental reflectance spectra agree with the $f$ value determined from the SEM image (Fig. 2(a)) within the uncertainty.

Figs. 3(c) and 3(d) present reflectance spectra from samples with $d_{1}=10 \mathrm{~nm}$ and $d_{2}=172$ and $320 \mathrm{~nm}$, respectively. Peaks are significantly broadened and red-shifted compared to the $d_{1}=20 \mathrm{~nm}$ data by much more than predicted by theory based on bulk permittivity, while the decrease in peak absorption is much less. However, EMA permittivity values from Eq. (4) with $f$ values as indicated in the plots give good match between theory and experiment. These fit values for $f$ agree with the value determined from image analysis (Fig. 2(b)) within the uncertainty, even though this uncertainty is smaller for this film.

For the sample with $d_{1}=20 \mathrm{~nm}$ and $d_{2}=320 \mathrm{~nm}$, the fundamental is at $1.14 \mu \mathrm{m}$. Its $93 \%$ peak absorption is maintained when $d_{1}$ is halved, but the wavelength range over which the fundamental absorption exceeds $90 \%$ becomes $50 \times$ broader. For the sample with $d_{1}=20 \mathrm{~nm}$ and $d_{2}=203 \mathrm{~nm}$, the experimental fundamental absorption at $0.81 \mu \mathrm{m}$ wavelength is $97 \%$ deep.

Fig. 4 presents angle dependent reflectance spectra with TE and TM polarizations for two of our samples. In comparison with Fig. 3, the spectral range is somewhat shorter on the long wave side due to the limitations of the different spectrometer used. Resonances are observed to red shift with increasing angle. The degree of agreement between theory and experiment is comparable to that in Fig. 3. As in that case, the single parameter used to match the theory to the experiment was the gold volume fraction $f$. It was necessary to treat the apparent gold fraction $f$ as being angle dependent. This parameter is varied manually until the shape and position of the resonances simultaneously agreed as closely as possible to the experimental result, as judged by eye.

The $f$ values that gave the best fit are plotted vs angle in Fig. 5 and compared to curves obtained from Eq. (5). The gap sizes $a$ were determined from Image J to be 37 and $13 \mathrm{~nm}$ for $\mathrm{d}_{1}=10$ and $20 \mathrm{~nm}$, respectively. The normal-incidence $f_{0}$ values used in Eq. (5) were those determined from the SEM image, namely, 0.54 and 0.85 , respectively. For the $20 \mathrm{~nm}$ thick top-layer, the very simple model for apparent gold fraction appears to work fairly well. At the highest angle of incidence, the apparent volume fraction is nearly unity, so that the permittivity approaches that of bulk gold.

For the $10 \mathrm{~nm}$ thick top-layer, the model curve is $2 \%-10 \%$ higher than the best-fit values. Perhaps here the neglected semitransparency of the gold becomes more

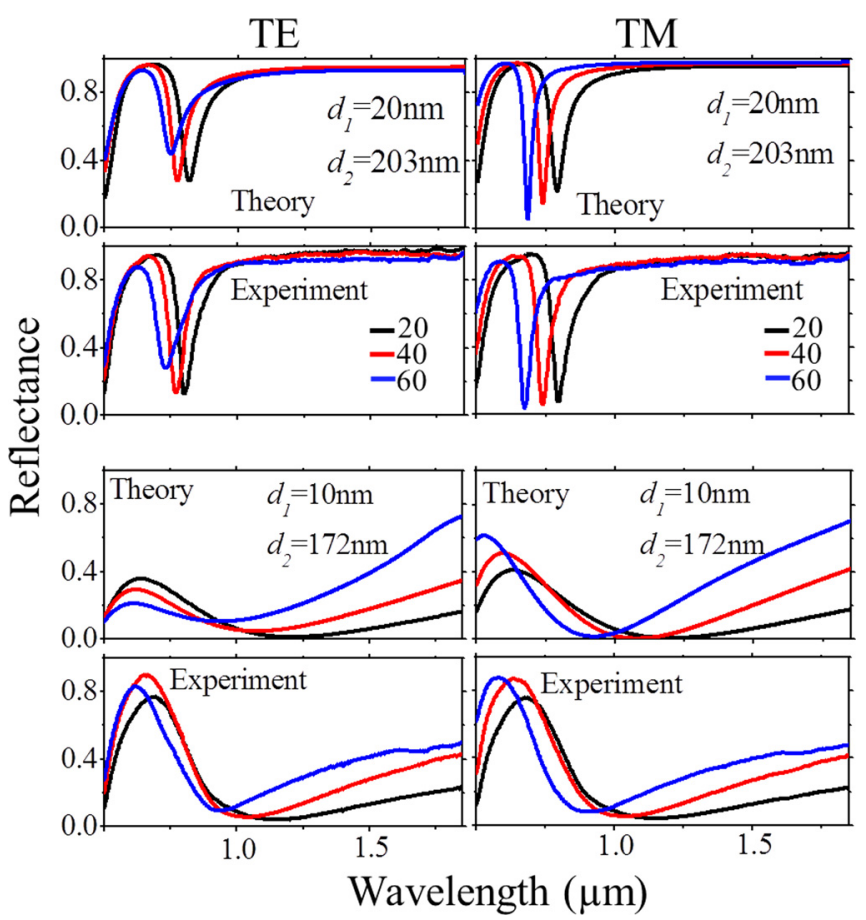

FIG. 4. Angle- and polarization-dependent reflectance spectra. The top (bottom) four plots correspond to thicknesses $d_{1}=20 \mathrm{~nm}$ and $d_{2}=203 \mathrm{~nm}$ $\left(d_{1}=10 \mathrm{~nm}, d_{2}=172 \mathrm{~nm}\right)$. The left (right) column of plots is for TE (TM) polarization. Theory and experiment are as indicated. All resonances shift to longer wavelength as angle of incidence is increased in the sequence $20^{\circ}$, $40^{\circ}$, and $60^{\circ}$.

important. Alternatively, the difference might be attributed to uncertainty in measured film parameters. For instance, by increasing the experimental value of the gap size $a$ by just a few percent, the curve shifts downward sufficiently to considerably improve the agreement.

Numerical finite-difference time-domain (FDTD) method, informed by 3D microstructure from AFM, might possibly provide a more accurate agreement with experiment and improved intuitive understanding. However, typical AFM tips would have difficulty navigating the deep narrow gaps in our surface films, ${ }^{24}$ so that obtaining accurate $3 \mathrm{D}$ morphology would be a challenge. Indeed, AFM measurements on our samples show height histograms with a single peak having a width that indicates a surface roughness of $\sim 2 \mathrm{~nm}$,

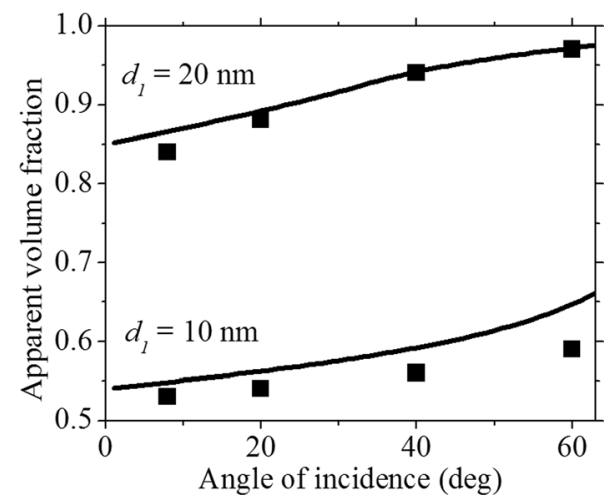

FIG. 5. Apparent gold volume fraction as function of incidence angle. Calculated values are presented by solid lines. Values used to fit the experimental reflectance spectra are given as symbols. 
which is the same as that of the commercial polished $\mathrm{Si}$ substrate.

Rapid design is well served by simple analytic models. All wavelengths considered are at least $10 \times$ longer than any microstructure, so we are clearly in the regime of macroscopic electrodynamics in which optical properties are determined by an appropriately averaged permittivity. The simple effective permittivity described here, with the resonance theory of Shu et al., predicts with remarkable accuracy the positions, widths, and peak strengths of the absorption resonances. However, the model tends to over-estimate the absorption between the resonances.

It would be very interesting and useful for applications to extrapolate the optical Salisbury screen to mid- and longwave infrared wavelengths. Such might use (e.g.) dielectric films of infrared-transparent glass such as AsSe. ${ }^{25}$ Such an IR effort would be timely, as suggested by the recent demonstrations of the two-dimensional thin-film total absorbers $n^{+} \mathrm{Si}$ on sapphire, ${ }^{26}$ heated $\mathrm{VO}_{2}$ on sapphire, ${ }^{27}$ and $\mathrm{Ge}$ on $\mathrm{n}^{+} \mathrm{Si}^{28}$

\section{SUMMARY}

In summary, a strongly absorbing optical Salisbury screen, based on metal-dielectric-metal resonator, has been experimentally demonstrated at visible and near-IR wavelengths. Maximum absorption was in the range 93\%-97\% for all resonances. Experiments confirm the theory of Shu et al., ${ }^{17}$ which we have successfully extended to the case of discontinuous top metal films by using permittivity values from effective medium approximation with input from microscopy. These absorbers require no in-plane patterning, yet give performance and design-tuning of resonance position and width comparable to much more sophisticated metamaterial absorbers. Hence, low cost fabrication over arbitrarily large areas may be achieved by wet chemical methods, in principle, i.e., as a wavelength-selective, ultra-black "paint." Potential applications include cloaking of (e.g.) military targets that might otherwise be susceptible to laser-marked guided munitions.

\section{ACKNOWLEDGMENTS}

This work was supported in part by an award from the Florida High Technology Corridor (I-4) program. We thank Ryuichi Tsuchikawa for assistance with the AFM measurements.
${ }^{1}$ N. I. Landy, S. Sajuyigbe, J. J. Mock, D. R. Smith, and W. J. Padilla, Phys. Rev. Lett. 100, 207402 (2008).

${ }^{2}$ J. Hao, L. Zhou, and M. Qiu, Phys. Rev. B 83, 165107 (2011).

${ }^{3}$ M. Diem, T. Koschny, and C. M. Soukoulis, Phys. Rev. B 79, 033101 (2009).

${ }^{4}$ N. Liu, M. Mesch, T. Weiss, M. Hentschel, and H. Giessen, Nano Lett. 10, $2342(2010)$.

${ }^{5}$ J. Hao, J. Wang, X. L. Liu, W. J. Padilla, L. Zhou, and M. Qiu, Appl. Phys. Lett. 96, 251104 (2010).

${ }^{6}$ J. Hendrickson, J. Guo, B. Zhang, W. Buchwald, and R. Soref, Opt. Lett. 37, 371 (2012).

${ }^{7}$ J. Wang, Y. Chen, J. Hao, M. Yan, and M. Qiu, J. Appl. Phys. 109, 074510 (2011).

${ }^{8}$ K. Aydin, V. E. Ferry, R. M. Briggs, and H. A. Atwater, Nat. Commun. 2, 517 (2011).

${ }^{9}$ K. B. Alici, A. B. Turhan, C. M. Soukoulis, and E. Ozbay, Opt. Express 19, 14260 (2011).

${ }^{10}$ M. K. Hedayati, M. Javaherirahim, B. Mozooni, R. Abdelaziz, A. Tavassolizadeh, V. S. K. Chakravadhanula, V. Zaporojtchenko, T. Strunkus, F. Faupel, and M. Elbahri, Adv. Mater. 23, 5410 (2011).

${ }^{11}$ H. Tao, N. I. Landy, C. M. Bingham, X. Zhang, R. D. Averitt, and W. J. Padilla, Opt. Express 16, 7181 (2008).

${ }^{12}$ J. Nath, D. Maukonen, E. Smith, P. Figueiredo, G. Zummo, D. Panjwani, R. E. Peale, G. Boreman, J. W. Cleary, and K. Eyink, Proc. SPIE 8704, 127 (2013).

${ }^{13}$ H. Tao, C. M. Bingham, A. C. Strikwerda, D. Pilon, D. Shrekenhamer, N. I. Landy, K. Fan, X. Zhang, W. J. Padilla, and R. D. Averitt, Phys. Rev. B 78, 241103 (2008).

${ }^{14}$ X. Wang, C. Luo, G. Hong, and X. Zhao, Appl. Phys. Lett. 102, 091902 (2013).

${ }^{15}$ W. W. Salisbury, U.S. patent 2,599,944 (1952).

${ }^{16}$ B. A. Munk, Frequency Selective Surfaces: Theory and Design (John Wiley \& Sons, 2005).

${ }^{17}$ S. Shu, Z. Li, and Y. Y. Li, Opt. Express 21, 25307 (2013).

${ }^{18}$ J. C. M. Garnett, Philos. Trans. R. Soc. London 203, 385 (1904); 205, 237 (1906).

${ }^{19}$ D. A. G. Bruggeman, Ann. Phys. (Leipzig) 416, 636 (1935).

${ }^{20}$ X. C. Zeng, D. J. Bergman, P. M. Hui, and D. Stroud, Phys. Rev. B 38, 10970 (1988).

${ }^{21}$ L. D. Landau, E. M. Lifshitz, and L. P. Pitaevskii, Electrodynamics of Continuous Media, 2nd ed. (Elsevier Butterworth Heineman, 1984), Sec. 80

${ }^{22}$ E. D. Palik, Handbook of Optical Constants of Solids, Academic Press Handbook Series (Academic Press, New York, 1985).

${ }^{23}$ T. J. Collins, Biotechniques 43, S25 (2007).

${ }^{24}$ R. E. Peale, O. Lopatiuk, J. Cleary, S. Santos, J. Henderson, D. Clark, L. Chernyak, T. A. Winningham, E. Del Barco, H. Heinrich, and W. R. Buchwald, J. Opt. Soc. Am. B 25, 1708 (2008).

${ }^{25}$ J. Nath, D. Panjwani, D. Maukonen, R. E. Peale, J. D. Musgraves, P. Wachtel, and J. McKinley, Proc. SPIE 9085, 7 (2014).

${ }^{26}$ J. W. Cleary, R. Soref, and J. R. Hendrickson, Opt. Express 21, 19363 (2013).

${ }^{27}$ M. A. Kats, D. Sharma, J. Lin, P. Genevet, R. Blanchard, Z. Yang, M. M. Qazilbash, D. N. Basov, S. Ramanathan, and F. Capasso, Appl. Phys. Lett. 101, 221101 (2012).

${ }^{28}$ W. Streyer, S. Law, G. Rooney, T. Jacobs, and D. Wasserman, Opt. Express 21, 9113 (2013). 